\title{
Generalization of the Euler Angles
}

\author{
Malcolm D. Shuster ${ }^{1}$ and F. Landis Markley ${ }^{2}$
}

\begin{abstract}
It is shown that the Euler angles can be generalized to axes other than members of an orthonormal triad. As first shown by Davenport, the three generalized Euler axes, hereafter: Davenport axes, must still satisfy the constraint that the first two and the last two axes be mutually perpendicular if these axes are to define a universal set of attitude parameters. Expressions are given which relate the generalized Euler angles, hereafter: Davenport angles, to the 3-1-3 Euler angles of an associated direction-cosine matrix. The computation of the Davenport angles from the attitude matrix and their kinematic equation are presented. The present work offers a more direct development of the Davenport angles than Davenport's original publication and offers additional results.
\end{abstract}

\section{Introduction}

The Euler angles [ $1-4]$ are defined as the angles of a sequence of three rotations

$$
R\left(\hat{\mathbf{n}}_{1}, \hat{\mathbf{n}}_{2}^{\prime}, \hat{\mathbf{n}}_{3}^{\prime \prime} ; \varphi, \vartheta, \psi\right) \equiv R\left(\hat{\mathbf{n}}_{3}^{\prime \prime}, \psi\right) R\left(\hat{\mathbf{n}}_{2}^{\prime}, \vartheta\right) R\left(\hat{\mathbf{n}}_{1}, \varphi\right)
$$

which represent the attitude, in particular, the attitude of a rigid body. Here, $\hat{\mathbf{n}}_{1}$, $\hat{\mathbf{n}}_{2}^{\prime}$, and $\hat{\mathbf{n}}_{3}^{\prime \prime}$ are selected from the set $\{\hat{\mathbf{1}}, \hat{\mathbf{2}}, \hat{\mathbf{3}}\}$, where

$$
\hat{\mathbf{1}} \equiv\left[\begin{array}{l}
1 \\
0 \\
0
\end{array}\right], \quad \hat{\mathbf{2}} \equiv\left[\begin{array}{l}
0 \\
1 \\
0
\end{array}\right], \quad \text { and } \quad \hat{\mathbf{3}} \equiv\left[\begin{array}{l}
0 \\
0 \\
1
\end{array}\right]
$$

In general, we denote column vectors by bold sans serif letters. A caret here denotes a unit column vector. The primes denote that the column vectors are each representations with respect to a different abstract basis (as seen by an inertial observer). In this case, the basis is the current basis of the body-fixed coordinate system, which changes (from an inertial point of view) as the body rotates.

\footnotetext{
${ }^{1}$ Acme Spacecraft Company, 13017 Wisteria Drive, Box 328, Germantown, Maryland 20874.

email: m.shuster@ieee.org.

${ }^{2}$ Aerospace Engineer, Guidance, Navigation and Control Systems Engineering Branch, Code 571, NASA

Goddard Space Flight Center, Greenbelt, MD 20771. email: landis.markley@.gsfc.nasa.gov.
} 
In order that the representation in terms of the Euler angles have the required three degrees of freedom, we must further stipulate that

$$
\hat{\mathbf{n}}_{1} \neq \hat{\mathbf{n}}_{2}^{\prime} \text { and } \hat{\mathbf{n}}_{2}^{\prime} \neq \hat{\mathbf{n}}_{3}^{\prime \prime} \text {. }
$$

Given this restriction, there are twelve possible sets of Euler angles: six symmetric sets, whose labels are written as

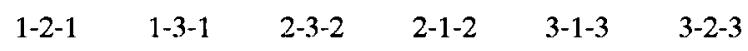

and six asymmetric sets, designated by

$$
1-2-3 \quad 1-3-2 \quad 2-3-1 \quad 2-1-3 \quad 3-1-2 \quad 3-2-1
$$

In each label the first (leftmost) integer denotes the first rotation axis. For example, the 1-3-2 set of Euler angles correspond to $\hat{\mathbf{n}}_{1}=\hat{\mathbf{1}}, \hat{\mathbf{n}}_{2}^{\prime}=\hat{\mathbf{3}}$, and $\hat{\mathbf{n}}_{3}^{\prime \prime}=\hat{\mathbf{2}}$. The asymmetric sets have been called variously Cardan angles, Bryant angles and Tait angles.

Regarded as the representation of body-fixed axes, the Euler axes are generally chosen from a right-hand orthonormal triad of column vectors. That this orthonormal triad need not be limited to the representations of the body coordinate axes with respect to themselves (that is, to the set $\{\hat{\mathbf{1}}, \hat{\mathbf{2}}, \hat{\mathbf{3}}\}$ ) should be obvious but will be demonstrated rigorously below. What we wish to know in particular is whether it is possible to construct a representation of the attitude in terms of Euler rotations about three arbitrary non-orthogonal axes as seen from the body-frame.

In the present note we prove that a universal representation of the attitude in terms of three consecutive Euler rotations about arbitrary non-orthogonal axes is not possible. However, we shall show that an cxtension of the definition of the Euler angles does indeed exist. The Euler angles for those sets of Euler axes which accommodate the representation of any attitude we shall refer to as universal attitude parameters, or as a universal representation, in order to distinguish them from the angles about three axes for which some attitudes cannot be represented.

\section{Universality of the Conventional Euler Angles}

To the best of our knowledge, all texts present formulas for extracting the conventional Euler angles from the rotation matrix, but none demonstrate rigorously that the Euler angles can represent an arbitrary rotation matrix. The proof is quite simple, and we offer it here.

We particularize our discussion to the 3-1-3 set of Euler angles. We shall show later in this report that any of the remaining eleven sets of conventional Euler angles can be obtained from the formula for extracting the 3-1-3 Euler angles. Hence, it the first step will be to prove that the 3-1-3 Euler angles are a universal parameterization of the attitude.

The explicit evaluation of equation (1) for $\hat{\mathbf{n}}_{1}=\hat{\mathbf{3}}, \hat{\mathbf{n}}_{2}=\hat{\mathbf{1}}$, and $\hat{\mathbf{n}}_{3}=\hat{\mathbf{3}}$ yields $^{3}$

$$
\begin{aligned}
& R_{313}(\varphi, \vartheta, \psi) \equiv R(\hat{\mathbf{3}}, \psi) R(\hat{\mathbf{1}}, \vartheta) R(\hat{\mathbf{3}}, \varphi) \\
& =\left[\begin{array}{ccc}
c \psi c \varphi-s \psi c \vartheta s \varphi & c \psi s \varphi+s \psi c \vartheta c \varphi & s \psi s \vartheta \\
-s \psi c \varphi-c \psi c \vartheta s \varphi & -s \psi s \varphi+c \psi c \vartheta c \varphi & c \psi s \vartheta \\
s \vartheta s \varphi & -s \vartheta c \varphi & c \vartheta
\end{array}\right]
\end{aligned}
$$

${ }^{3}$ Henceforth, we shall discard the primes on the Euler-axis representations with the understanding that these are always with respect to current body axes. 
where $c \varphi=\cos \varphi, s \varphi=\sin \varphi$, etc., and we have written the axis indices as subscripts on $R$. The elements of the attitude matrix (direction-cosine matrix) are the components of the initial body axes with respect to the final body axes. In order to demonstrate that the parameterization of $R_{313}$ can realize any proper orthogonal matrix, it is sufficient to show that the representation of the coordinate axes of the initial coordinate system with respect to themselves (namely, $\hat{\mathbf{1}}, \hat{\mathbf{2}}$ and $\hat{\mathbf{3}}$ ) can be transformed into any other right-hand orthonormal triad of column vectors, i.e., the representation with respect to an arbitrary set of arbitrary right-hand orthonormal coordinate axes.

Thus, we cxamine first

$$
\hat{\mathbf{e}}_{3} \equiv R_{313}(\varphi, \vartheta, \psi) \hat{\mathbf{3}}=\left[\begin{array}{c}
\sin \vartheta \sin \psi \\
\sin \vartheta \cos \psi \\
\cos \vartheta
\end{array}\right]
$$

Clearly, $\vartheta$ and $(\pi / 2-\psi)$ are the spherical angles of an arbitrary unit vector. Hence, by a suitable choice of $\vartheta$ and $\psi, \hat{\mathbf{e}}_{3}$ can be made to coincide with an arbitrary unit column vector, in particular, the third member of the target right-hand orthonormal triad. We will assume that $\vartheta$ and $\psi$ have been so chosen.

Examine next

$$
\hat{\mathbf{e}}_{1} \equiv R_{313}(\varphi, \vartheta, \psi) \hat{\mathbf{1}}=\left[\begin{array}{c}
\cos \psi \cos \varphi-\cos \vartheta \sin \varphi \sin \psi \\
-\sin \psi \cos \varphi-\cos \vartheta \sin \varphi \cos \psi \\
\sin \vartheta \sin \varphi
\end{array}\right]
$$

This last column vector can be written as

$$
\hat{\mathbf{e}}_{1}=\cos \varphi \hat{\mathbf{u}}_{1}+\sin \varphi \hat{\mathbf{u}}_{2},
$$

where

$$
\hat{\mathbf{u}}_{1}=\left[\begin{array}{c}
\cos \psi \\
-\sin \psi \\
0
\end{array}\right], \quad \hat{\mathbf{u}}_{2}=\left[\begin{array}{c}
-\cos \vartheta \sin \psi \\
-\cos \vartheta \cos \psi \\
\sin \vartheta
\end{array}\right]
$$

Obviously, $\hat{\mathbf{u}}_{1}$ and $\hat{\mathbf{u}}_{2}$ are each unit column vectors and

$$
\hat{\mathbf{u}}_{1} \cdot \hat{\mathbf{e}}_{3}=\hat{\mathbf{u}}_{2} \cdot \hat{\mathbf{e}}_{3}=\hat{\mathbf{u}}_{1} \cdot \hat{\mathbf{u}}_{2}=0 \text {. }
$$

The vectors $\hat{\mathbf{u}}_{1}$ and $\hat{\mathbf{u}}_{2}$ span the plane perpendicular to $\hat{\mathbf{e}}_{3}$, and $\varphi$ can be chosen to generate any unit vector in that plane. Since the first member of the target right-hand orthonormal triad must lie in that plane, there is a value of $\varphi$ for which $\hat{\mathbf{e}}_{1}$ coincides with that unit column vector. Thus, for suitable choices of the three Euler angles, $R_{313}$ will transform $\hat{\mathbf{3}}$ and $\hat{\mathbf{1}}$ into any pair of mutually perpendicular unit column vectors.

Finally, because both the initial column vectors $\{\hat{\mathbf{1}}, \hat{\mathbf{2}}, \hat{\mathbf{3}}\})$ and the target column vectors $\left\{\hat{\mathbf{e}}_{1}, \hat{\mathbf{e}}_{2}, \hat{\mathbf{e}}_{3}\right\}$ are each right-hand orthonormal sets and $R_{313}(\varphi, \vartheta, \psi)$ is proper orthogonal by construction, it follows that

$$
\begin{aligned}
R_{313}(\varphi, \vartheta, \psi) \hat{\mathbf{2}} & =R_{313}(\varphi, \vartheta, \psi)(\hat{\mathbf{3}} \times \hat{\mathbf{1}}) \\
& =\left(R_{313}(\varphi, \vartheta, \psi) \hat{\mathbf{3}}\right) \times\left(R_{313}(\varphi, \vartheta, \psi) \hat{\mathbf{1}}\right) \\
& =\hat{\mathbf{e}}_{3} \times \hat{\mathbf{e}}_{1}=\hat{\mathbf{e}}_{2}
\end{aligned}
$$


which completes the proof. Therefore, the 3-1-3 Euler angles are a universal representation of the attitude. The proof can be repeated with minor modifications for any of the other eleven conventional sets of Euler angles, but the universality of these remaining sets will follow as an corollary of the result for the generalized Euler angles.

\section{Generalized Euler Angles (Davenport Angles)}

Consider a three-parameter rotation given by

$$
R\left(\hat{\mathbf{n}}_{1}, \hat{\mathbf{n}}_{2}, \hat{\mathbf{n}}_{3} ; \varphi, \vartheta, \psi\right) \equiv R\left(\hat{\mathbf{n}}_{3}, \psi\right) R\left(\hat{\mathbf{n}}_{2}, \vartheta\right) R\left(\hat{\mathbf{n}}_{1}, \varphi\right)
$$

where now, there are no restrictions on $\hat{\mathbf{n}}_{1}, \hat{\mathbf{n}}_{2}$, and $\hat{\mathbf{n}}_{3}$, except that

$$
\hat{\mathbf{n}}_{1} \neq \hat{\mathbf{n}}_{2} \text { and } \hat{\mathbf{n}}_{2} \neq \hat{\mathbf{n}}_{3} \text {. }
$$

Can any rotation be represented in this way? A necessary condition for this to be true is that any unit column vector can be transformed into any other unit column vector by this sequence of rotations. For the generalized Euler angles to be a universal representation of the attitude, this condition must be satisficd.

Consider now the transformation

$$
\hat{\mathbf{v}}=R\left(\hat{\mathbf{n}}_{1}, \hat{\mathbf{n}}_{2}, \hat{\mathbf{n}}_{3} ; \varphi, \vartheta, \psi\right) \hat{\mathbf{n}}_{1}
$$

Then

$$
\hat{\mathbf{n}}_{3} \cdot \hat{\mathbf{v}}=\hat{\mathbf{n}}_{3} \cdot R\left(\hat{\mathbf{n}}_{2}, \vartheta\right) \hat{\mathbf{n}}_{1}
$$

We shall show that equation (14) cannot be satisfied for arbitrary $\hat{\mathbf{n}}_{1}, \hat{\mathbf{n}}_{2}, \hat{\mathbf{n}}_{3}$, and $\hat{\mathbf{v}}$.

Writing Euler's formula as [3]

$$
R(\hat{\mathbf{n}}, \zeta)=I_{3 \times 3}+\sin \zeta[[\hat{\mathbf{n}}]]+(1-\cos \zeta)[[\hat{\mathbf{n}}]]^{2},
$$

with

$$
[[\mathbf{u}]\} \equiv\left[\begin{array}{ccc}
0 & u_{3} & -u_{2} \\
-u_{3} & 0 & u_{1} \\
u_{2} & -u_{1} & 0
\end{array}\right],
$$

it follows straightforwardly that the condition on $\vartheta$ becomes

$$
\begin{aligned}
\hat{\mathbf{n}}_{3} \cdot \hat{\mathbf{v}}=\left(\hat{\mathbf{n}}_{3} \cdot \hat{\mathbf{n}}_{1}\right)+\hat{\mathbf{n}}_{3} \cdot\left(\hat{\mathbf{n}}_{2} \times\left(\hat{\mathbf{n}}_{2} \times \hat{\mathbf{n}}_{1}\right)\right)-\sin \vartheta\left(\hat{\mathbf{n}}_{3} \cdot\left(\hat{\mathbf{n}}_{2} \times \hat{\mathbf{n}}_{1}\right)\right) \\
-\cos \vartheta\left(\hat{\mathbf{n}}_{3} \cdot\left(\hat{\mathbf{n}}_{2} \times\left(\hat{\mathbf{n}}_{2} \times \hat{\mathbf{n}}_{1}\right)\right)\right) .
\end{aligned}
$$

Define now

$$
B \cos \alpha \equiv-\hat{\mathbf{n}}_{3} \cdot\left(\hat{\mathbf{n}}_{2} \times\left(\hat{\mathbf{n}}_{2} \times \hat{\mathbf{n}}_{1}\right)\right) \quad \text { and } B \sin \alpha \equiv-\hat{\mathbf{n}}_{3} \cdot\left(\hat{\mathbf{n}}_{2} \times \hat{\mathbf{n}}_{1}\right),
$$

or, equivalently,

$$
\begin{aligned}
& \alpha=\arctan _{2}\left[\hat{\mathbf{n}}_{1} \cdot\left(\hat{\mathbf{n}}_{2} \times \hat{\mathbf{n}}_{3}\right),-\hat{\mathbf{n}}_{1} \cdot\left(\hat{\mathbf{n}}_{2} \times\left(\hat{\mathbf{n}}_{2} \times \hat{\mathbf{n}}_{3}\right)\right)\right], \\
& B=\sqrt{\left[\hat{\mathbf{n}}_{3} \cdot\left(\hat{\mathbf{n}}_{2} \times \hat{\mathbf{n}}_{1}\right)\right]^{2}+\left[\hat{\mathbf{n}}_{3} \cdot\left(\hat{\mathbf{n}}_{2} \times\left(\hat{\mathbf{n}}_{2} \times \hat{\mathbf{n}}_{1}\right)\right)\right]^{2}}>0,
\end{aligned}
$$


where $\arctan _{2}(y, x)$ is the function which yields the arc tangent of $y / x$ in the correct quadrant. This corresponds to the function ATAN2 in the FORTRAN programming language. In terms of these new variables, equation (17) becomes

$$
\hat{\mathbf{n}}_{3} \cdot \hat{\mathbf{v}}=\beta+B \cos (\vartheta-\alpha),
$$

where

$$
\beta \equiv\left(\hat{\mathbf{n}}_{3} \cdot \hat{\mathbf{n}}_{1}\right)+\hat{\mathbf{n}}_{3} \cdot\left(\hat{\mathbf{n}}_{2} \times\left(\hat{\mathbf{n}}_{2} \times \hat{\mathbf{n}}_{1}\right)\right)=\left(\hat{\mathbf{n}}_{3} \cdot \hat{\mathbf{n}}_{2}\right)\left(\hat{\mathbf{n}}_{2} \cdot \hat{\mathbf{n}}_{1}\right) .
$$

The right member of equation (20) can assume any value between $\beta-B$ and $\beta+B$. Therefore, a solution will exist for $\vartheta$ if and only if

$$
\beta+B \geq \hat{\mathbf{n}}_{3} \cdot \hat{\mathbf{v}} \geq \beta-B
$$

However, since $\hat{\mathbf{n}}_{3} \cdot \hat{\mathbf{v}}$ can assume any value between -1 and +1 , it follows from equation (22) that $\beta$ and $B$ must satisfy

$$
B \geq 1-\beta \text { and } B \geq 1+\beta .
$$

Thus, we require that

$$
B \geq 1+|\beta|
$$

On the other hand, defining

$$
\mathbf{u} \equiv \hat{\mathbf{n}}_{2} \times \hat{\mathbf{n}}_{1}
$$

it follows that

$$
\begin{aligned}
B^{2} & =\left[\hat{\mathbf{n}}_{3} \cdot \mathbf{u}\right]^{2}+\left[\hat{\mathbf{n}}_{3} \cdot\left(\hat{\mathbf{n}}_{2} \times \mathbf{u}\right)\right]^{2} \\
& =|\mathbf{u}|^{2} \hat{\mathbf{n}}_{3}^{T}\left\{\hat{\mathbf{u}} \hat{\mathbf{u}}^{T}+\left(\hat{\mathbf{n}}_{2} \times \hat{\mathbf{u}}\right)\left(\hat{\mathbf{n}}_{2} \times \hat{\mathbf{u}}\right)^{T}\right\} \hat{\mathbf{n}}_{3} .
\end{aligned}
$$

Now, $\hat{\mathbf{n}}_{2}$ and $\hat{\mathbf{u}}$ are orthogonal. Hence, $\hat{\mathbf{n}}_{2}$, $\hat{\mathbf{u}}$, and $\hat{\mathbf{n}}_{2} \times \hat{\mathbf{u}}$, form an orthonormal triad, and therefore

$$
\hat{\mathbf{n}}_{2} \hat{\mathbf{n}}_{2}^{T}+\hat{\mathbf{u}} \hat{\mathbf{u}}^{T}+\left(\hat{\mathbf{n}}_{2} \times \hat{\mathbf{u}}\right)\left(\hat{\mathbf{n}}_{2} \times \hat{\mathbf{u}}\right)^{T}=I_{3 \times 3}
$$

It follows that

$$
B^{2}=|\mathbf{u}|^{2} \hat{\mathbf{n}}_{3}^{T}\left[I_{3 \times 3}-\hat{\mathbf{n}}_{2} \hat{\mathbf{n}}_{2}^{T}\right] \hat{\mathbf{n}}_{3}=|\mathbf{u}|^{2}\left[1-\left(\hat{\mathbf{n}}_{3} \cdot \hat{\mathbf{n}}_{2}\right)^{2}\right]=\left|\hat{\mathbf{n}}_{3} \times \hat{\mathbf{n}}_{2}\right|^{2}\left|\hat{\mathbf{n}}_{2} \times \hat{\mathbf{n}}_{1}\right|^{2} .
$$

We have thus

$$
\beta=\left(\hat{\mathbf{n}}_{3} \cdot \hat{\mathbf{n}}_{2}\right)\left(\hat{\mathbf{n}}_{2} \cdot \hat{\mathbf{n}}_{1}\right), \quad B=\left|\hat{\mathbf{n}}_{3} \times \hat{\mathbf{n}}_{2}\right|\left|\hat{\mathbf{n}}_{2} \times \hat{\mathbf{n}}_{1}\right| .
$$

Equations (24) and (29) can be satisfied simultaneously if and only if

$$
B=1 \text { and } \beta=0 .
$$

Hence, we require that

$$
\hat{\mathbf{n}}_{1} \perp \hat{\mathbf{n}}_{2} \text { and } \hat{\mathbf{n}}_{2} \perp \hat{\mathbf{n}}_{3}
$$

as a necessary condition that the generalized Euler angles be able to represent an arbitrary attitude. Equations (31) were first discovered by Paul Davenport [5], who 
also proved sufficiency, as we shall below. For this reason we will refer henceforth to the generalized Euler angles as the Davenport angles.

Equation (31) is a less restrictive condition than the generalization of equations (2) and (3) to an arbitrary right-hand orthonormal triad, which would have required further that $\left(\hat{\mathbf{n}}_{1} \cdot \hat{\mathbf{n}}_{3}\right)$ be either 0 or 1 . The additional degree of freedom allowed by equation (31) is the angle between $\hat{\mathbf{n}}_{1}$ and $\hat{\mathbf{n}}_{3}$.

We shall now prove that the condition expressed by equation (31) is sufficient for the set of Davenport axes to permit a universal parameterization of the attitude in terms of Davenport angles. Let us write

$$
\hat{\mathbf{n}}_{3}=R\left(\hat{\mathbf{n}}_{2}, \lambda\right) \hat{\mathbf{n}}_{1},
$$

where $\hat{\mathbf{n}}_{1}$ and $\hat{\mathbf{n}}_{2}$ are orthogonal. Then,

$$
\hat{\mathbf{n}}_{3}=\cos \lambda \hat{\mathbf{n}}_{1}-\sin \lambda\left(\hat{\mathbf{n}}_{2} \times \hat{\mathbf{n}}_{1}\right), \quad-\pi<\lambda \leq \pi,
$$

which clearly satisfies $\hat{\mathbf{n}}_{3} \perp \hat{\mathbf{n}}_{2}$ and is the most general column vector satisfying this condition. $\lambda$ is the angle from $\hat{\mathbf{n}}_{1}$ to $\hat{\mathbf{n}}_{3}$, defined to be positive in the counter-clockwise direction about $\hat{\mathbf{n}}_{2}$. The symmetric sequences of Euler angles correspond to $\lambda=0$, while the asymmetric sequences correspond to either $\lambda=\pi / 2$ or $\lambda=-\pi / 2$. If $\hat{\mathbf{n}}_{2}$ is the cyclic follower of $\hat{\mathbf{n}}_{1}$, then $\lambda=\pi / 2$. If it is the anticyclic follower, then $\lambda=-\pi / 2$.

It follows [3] that

$$
\begin{aligned}
R\left(\hat{\mathbf{n}}_{3}, \psi\right) & R\left(\hat{\mathbf{n}}_{2}, \vartheta\right) R\left(\hat{\mathbf{n}}_{1}, \varphi\right) \\
= & R\left(R\left(\hat{\mathbf{n}}_{2}, \lambda\right) \hat{\mathbf{n}}_{1}, \psi\right) R\left(\hat{\mathbf{n}}_{2}, \vartheta\right) R\left(\hat{\mathbf{n}}_{1}, \varphi\right) \\
& =R\left(\hat{\mathbf{n}}_{2}, \lambda\right) R\left(\hat{\mathbf{n}}_{1}, \psi\right) R^{T}\left(\hat{\mathbf{n}}_{2}, \lambda\right) R\left(\hat{\mathbf{n}}_{2}, \vartheta\right) R\left(\hat{\mathbf{n}}_{1}, \varphi\right) \\
& =R\left(\hat{\mathbf{n}}_{2}, \lambda\right) R\left(\hat{\mathbf{n}}_{1}, \hat{\mathbf{n}}_{2}, \hat{\mathbf{n}}_{1} ; \varphi, \vartheta^{\prime}, \psi\right)
\end{aligned}
$$

where

$$
\vartheta^{\prime}=\vartheta-\lambda \text {. }
$$

If $A$ is an arbitrary proper orthogonal matrix, then we wish to find angles $\left(\varphi, \vartheta^{\prime}, \psi\right)$ which satisfy

$$
A=R\left(\hat{\mathbf{n}}_{1}, \hat{\mathbf{n}}_{2}, \hat{\mathbf{n}}_{3} ; \varphi, \vartheta, \psi\right)=R\left(\hat{\mathbf{n}}_{2}, \lambda\right) R\left(\hat{\mathbf{n}}_{1}, \hat{\mathbf{n}}_{2}, \hat{\mathbf{n}}_{1} ; \varphi, \vartheta^{\prime}, \psi\right) .
$$

To show that this is possible, let $C$ be the proper orthogonal matrix which satisfies

$$
C \hat{\mathbf{n}}_{1}=\hat{\mathbf{3}}, \text { and } C \hat{\mathbf{n}}_{2}=\hat{\mathbf{1}} \text {. }
$$

Since $\hat{\mathbf{n}}_{1}$ and $\hat{\mathbf{n}}_{2}$ are orthogonal, the matrix $C$ exists and is given by

$$
C=\left[\begin{array}{lll}
\hat{\mathbf{n}}_{2} & \left(\hat{\mathbf{n}}_{1} \times \hat{\mathbf{n}}_{2}\right) & \hat{\mathbf{n}}_{1}
\end{array}\right]^{T},
$$

where the expression for $C$ is given as the transpose of a proper orthogonal matrix labeled by its column vectors. It follows from equations (36) and (38) that the triplet $\left(\varphi, \vartheta^{\prime}, \psi\right)$ must satisfy

$$
R\left(\hat{\mathbf{3}}, \hat{\mathbf{1}}, \hat{\mathbf{3}} ; \varphi, \vartheta^{\prime}, \psi\right)=R^{T}(\hat{\mathbf{1}}, \lambda) C A C^{T} .
$$


The triplet $\left(\varphi, \vartheta^{\prime}, \psi\right)$ is now simply the 3-1-3 set of Euler angles representing the matrix which is the right member of equation (39). Since the 3-1-3 Euler angles have been shown to be a universal representation of the attitude, we know that a solution always exists. This proves the sufficiency of equations (31). Q.E.D.

As a by-product of our proof we have that

$$
R\left(\hat{\mathbf{n}}_{1}, \hat{\mathbf{n}}_{2}, \hat{\mathbf{n}}_{3} ; \varphi, \vartheta, \psi\right)=C^{T} R(\hat{\mathbf{1}}, \lambda) R(\hat{\mathbf{3}}, \hat{\mathbf{1}}, \hat{\mathbf{3}} ; \varphi, \vartheta-\lambda, \psi) C
$$

for any set of axes satisfying equation (31) and $C$ given by equation (38). As an immediate corollary we have that any of the twelve conventional sets of Euler angles is a universal attitude representation. For a suitable choice of $\lambda$ and $C$, expressions similar to equation (40) can be obtained for all twelve sets of conventional Euler angles.

Recall that $\lambda$ is a function solcly of the axes.

$$
\lambda=\arctan _{2}\left(\left(\hat{\mathbf{n}}_{1} \times \hat{\mathbf{n}}_{2}\right) \cdot \hat{\mathbf{n}}_{3}, \hat{\mathbf{n}}_{1} \cdot \hat{\mathbf{n}}_{3}\right) .
$$

This formula also assumes that equation (31) holds.

We remark that while the three Davenport axes must satisfy equation (31) in order for the representation to be universal, the parameterization of the attitude can still be useful when only equation (12) is satisfied, if one knows a priori that the axes permit a realization of the attitude matrix for the values of interest.

\section{Extracting the Davenport Angles}

To determine the Davenport angles from a given direction-cosine matrix we note the relationships

$$
\begin{aligned}
\hat{\mathbf{n}}_{3}^{T} A \hat{\mathbf{n}}_{1} & =\cos (\vartheta-\lambda), \\
\hat{\mathbf{n}}_{2}^{T} A \hat{\mathbf{n}}_{1} & =\sin (\vartheta-\lambda) \sin \psi, \\
\left(\hat{\mathbf{n}}_{2} \times \hat{\mathbf{n}}_{3}\right)^{T} A \hat{\mathbf{n}}_{1} & =-\sin (\vartheta-\lambda) \cos \psi, \\
\hat{\mathbf{n}}_{3}^{T} A \hat{\mathbf{n}}_{2} & =\sin (\vartheta-\lambda) \sin \varphi, \\
\hat{\mathbf{n}}_{3}^{T} A\left(\hat{\mathbf{n}}_{1} \times \hat{\mathbf{n}}_{2}\right) & =-\sin (\vartheta-\lambda) \cos \varphi .
\end{aligned}
$$

Except for the fact that $\vartheta$ has been replaced by $\vartheta-\lambda$, the right members of equations (42) are each identical within a sign to the elements of the directioncosine matrix given in equation (4).

From equation (42a) we have immcdiately

$$
\vartheta=\lambda+\arccos \left(\hat{\mathbf{n}}_{3}^{T} A \hat{\mathbf{n}}_{1}\right),
$$

If we choose the principal value of the arc cosine, then $\vartheta$ will be single valued and lie in the range

$$
\lambda \leq \vartheta \leq \lambda+\pi .
$$

For $\lambda<\vartheta<\lambda+\pi$, so that $\sin (\vartheta-\lambda)>0$ the two remaining Davenport angles are given by

$$
\begin{aligned}
& \varphi=\arctan _{2}\left[\hat{\mathbf{n}}_{3}^{T} A \hat{\mathbf{n}}_{2},-\hat{\mathbf{n}}_{3}^{T} A\left(\hat{\mathbf{n}}_{1} \times \hat{\mathbf{n}}_{2}\right)\right] \\
& \psi=\arctan _{2}\left[\hat{\mathbf{n}}_{2}^{T} A \hat{\mathbf{n}}_{1},-\left(\hat{\mathbf{n}}_{2} \times \hat{\mathbf{n}}_{3}\right)^{T} A \hat{\mathbf{n}}_{1}\right] .
\end{aligned}
$$


The cases where $\sin (\vartheta-\lambda)=0$ are treated in the next section.

\section{Singularity of the Davenport Angles}

The representation in terms of Davenport angles must become singular when

$$
R\left(\hat{\mathbf{n}}_{2}, \vartheta\right) \hat{\mathbf{n}}_{1}= \pm \hat{\mathbf{n}}_{3}
$$

which follows from an examination of the resulting equations

$$
\begin{aligned}
R\left(\hat{\mathbf{n}}_{3}, \psi\right) & R\left(\hat{\mathbf{n}}_{2}, \vartheta\right) R\left(\hat{\mathbf{n}}_{1}, \varphi\right) \\
& =R\left(\hat{\mathbf{n}}_{3}, \psi\right) R\left(\hat{\mathbf{n}}_{2}, \vartheta\right) R\left(\hat{\mathbf{n}}_{1}, \varphi\right) R^{T}\left(\hat{\mathbf{n}}_{2}, \vartheta\right) R\left(\hat{\mathbf{n}}_{2}, \vartheta\right) \\
& =R\left(\hat{\mathbf{n}}_{3}, \psi\right) R\left( \pm \hat{\mathbf{n}}_{3}, \varphi\right) R\left(\hat{\mathbf{n}}_{2}, \vartheta\right) \\
& =R\left(\hat{\mathbf{n}}_{3}, \psi \pm \varphi\right) R\left(\hat{\mathbf{n}}_{2}, \vartheta\right) \\
& =R\left(\hat{\mathbf{n}}_{2}, \vartheta\right) R\left(\hat{\mathbf{n}}_{1}, \varphi \pm \psi\right)
\end{aligned}
$$

where we assumed equation (46) in going from equation (47a) to equation (47b). It is easy to see from equation (42a) that a singularity in the Davenport angles occurs when $\sin (\vartheta-\lambda)=0$.

Equations (45) are inappropriate for the computation of $\varphi$ and $\psi$ at a singularity, when $\vartheta-\lambda=0$ or $\pi$, because all of the arguments of the $\arctan _{2}$ functions will vanish. In that case we must turn to four other "elements" of the direction-cosine matrix, namely,

$$
\begin{aligned}
& \hat{\mathbf{n}}_{2}^{T} A\left(\hat{\mathbf{n}}_{1} \times \hat{\mathbf{n}}_{2}\right)=\sin \varphi \cos \psi+\cos \varphi \sin \psi \cos (\vartheta-\lambda) \equiv a, \\
&\left(\hat{\mathbf{n}}_{2} \times \hat{\mathbf{n}}_{3}\right)^{T} A \hat{\mathbf{n}}_{2}=\cos \varphi \sin \psi+\sin \varphi \cos \psi \cos (\vartheta-\lambda) \equiv b, \\
& \hat{\mathbf{n}}_{2}^{T} A \hat{\mathbf{n}}_{2}=\cos \varphi \cos \psi-\sin \varphi \sin \psi \cos (\vartheta-\lambda) \equiv c \\
&\left(\hat{\mathbf{n}}_{2} \times \hat{\mathbf{n}}_{3}\right)^{T} A\left(\hat{\mathbf{n}}_{1} \times \hat{\mathbf{n}}_{2}\right)=\sin \varphi \sin \psi-\cos \varphi \cos \psi \cos (\vartheta-\lambda) \equiv d
\end{aligned}
$$

From these relationships it follows that

$$
\begin{aligned}
& {[1 \pm \cos (\vartheta-\lambda)] \sin (\varphi \pm \psi)=a \pm b,} \\
& {[1 \pm \cos (\vartheta-\lambda)] \cos (\varphi \pm \psi)=c \mp d,}
\end{aligned}
$$

from which it follows that

$$
\begin{array}{lll}
\varphi+\psi=\arctan _{2}(a+b, c-d) & \text { for } & \cos (\vartheta-\lambda) \neq-1 \\
\varphi-\psi=\arctan _{2}(a-b, c+d) & \text { for } & \cos (\vartheta-\lambda) \neq+1
\end{array}
$$

These equations provide a more accurate means for calculating either $\varphi+\psi$ or $\varphi-\psi$ (but, unfortunately, not both) when the attitude is very close to a singularity than do cquations (45). 
The Davenport angles extracted by this procedure and that of the previous section will not always agree with the conventional Euler angles when a conventional Euleraxis set is used. This is due to trivial angular ambiguities of $2 \pi$ and to the two-fold ambiguity in the Davenport angles, which is related to the well-known two-fold ambiguity of the conventional Euler angles. The general relation for the two-fold ambiguity in the Davenport angles can be obtained from equation (40) and the similar relation for the 3-1-3 Euler angles, namely,

$$
R(\hat{\mathbf{3}}, \hat{\mathbf{i}}, \hat{\mathbf{3}} ; \varphi, \vartheta, \psi)=R(\hat{\mathbf{3}}, \hat{\mathbf{1}}, \hat{\mathbf{3}} ; \varphi+\pi,-\vartheta, \psi-\pi),
$$

with the result

$$
R\left(\hat{\mathbf{n}}_{1}, \hat{\mathbf{n}}_{2}, \hat{\mathbf{n}}_{3} ; \varphi, \vartheta, \psi\right)=R\left(\hat{\mathbf{n}}_{1}, \hat{\mathbf{n}}_{2}, \hat{\mathbf{n}}_{3} ; \varphi+\pi, 2 \lambda-\vartheta, \psi-\pi\right),
$$

which summarizes the result for the 3-1-3 Euler angles $(\lambda=0)$ and the 3-1-2 Euler angles $(\lambda=\pi / 2)$. The specification of the formulas of this section and the previous section to the extraction of the conventional Euler angles is left as an exercise for the reader.

\section{Kinematics}

The kinematic equation for the Davenport angles is identical to the general expressions for the conventional Euler angles. ${ }^{4}$ We may write [3]

$$
\boldsymbol{\omega}=\dot{\psi} \hat{\mathbf{n}}_{3}+\dot{\vartheta} R\left(\hat{\mathbf{n}}_{3}, \psi\right) \hat{\mathbf{n}}_{2}+\dot{\varphi} R\left(\hat{\mathbf{n}}_{3}, \psi\right) R\left(\hat{\mathbf{n}}_{2}, \vartheta\right) \hat{\mathbf{n}}_{1} .
$$

where $\omega$ is the body-referenced angular velocity vector. This may recast in the form

$$
\boldsymbol{\omega}=R\left(\hat{\mathbf{n}}_{3}, \psi\right) S\left(\hat{\mathbf{n}}_{1}, \hat{\mathbf{n}}_{2}, \hat{\mathbf{n}}_{3} ; \vartheta\right)\left[\begin{array}{c}
\dot{\varphi} \\
\dot{\vartheta} \\
\dot{\psi}
\end{array}\right] \equiv M(\varphi, \vartheta, \psi)\left[\begin{array}{c}
\dot{\varphi} \\
\dot{\vartheta} \\
\dot{\psi}
\end{array}\right],
$$

with $S\left(\hat{\mathbf{n}}_{1}, \hat{\mathbf{n}}_{2}, \hat{\mathbf{n}}_{3} ; \vartheta\right)$ represented in terms of column vectors as

$$
S\left(\hat{\mathbf{n}}_{1}, \hat{\mathbf{n}}_{2}, \hat{\mathbf{n}}_{3} ; \vartheta\right)=\left[\begin{array}{llllll}
R\left(\hat{\mathbf{n}}_{2}, \vartheta\right) & \hat{\mathbf{n}}_{1} & \mid & \hat{\mathbf{n}}_{2} & \mid & \hat{\mathbf{n}}_{3}
\end{array}\right] .
$$

Simple forms exist for the matrices $S\left(\hat{\mathbf{n}}_{1}, \hat{\mathbf{n}}_{2}, \hat{\mathbf{n}}_{3} ; \vartheta\right)$ and $M(\varphi, \vartheta, \psi)$ as explicit functions of the conventional Euler angles [4]. Those for the Davenport angles are more complicated.

More useful for simulation is the inverse of $M(\varphi, \vartheta, \psi)$, which satisfies

$$
\left[\begin{array}{c}
\dot{\varphi} \\
\dot{\vartheta} \\
\dot{\psi}
\end{array}\right]=M^{-1}(\varphi, \vartheta, \psi) \omega
$$

whence

$$
M^{-1}(\varphi, \vartheta, \psi)=S^{-1}\left(\hat{\mathbf{n}}_{1}, \hat{\mathbf{n}}_{2}, \hat{\mathbf{n}}_{3} ; \vartheta\right) R^{T}\left(\hat{\mathbf{n}}_{3}, \psi\right)
$$

with

$$
S^{-1}\left(\hat{\mathbf{n}}_{1}, \hat{\mathbf{n}}_{2}, \hat{\mathbf{n}}_{3} ; \vartheta\right)=\frac{1}{\left(R\left(\hat{\mathbf{n}}_{2}, \vartheta\right) \hat{\mathbf{n}}_{1}\right) \cdot\left(\hat{\mathbf{n}}_{2} \times \hat{\mathbf{n}}_{3}\right)}\left[\begin{array}{c}
{\left[\hat{\mathbf{n}}_{2} \times \hat{\mathbf{n}}_{3}\right]^{T}} \\
{\left[\hat{\mathbf{n}}_{3} \times R\left(\hat{\mathbf{n}}_{2}, \vartheta\right) \hat{\mathbf{n}}_{1}\right]^{T}} \\
{\left[\left(R\left(\hat{\mathbf{n}}_{2}, \vartheta\right) \hat{\mathbf{n}}_{1}\right) \times \hat{\mathbf{n}}_{2}\right]^{T}}
\end{array}\right]
$$

\footnotetext{
${ }^{4}$ The kinematic equation for the Davenport angles was also derived by Davenport [5].
} 
The right member of equation (58) can be reduced to

$$
\frac{1}{D}\left[\begin{array}{c}
{\left[\hat{\mathbf{n}}_{2} \times \hat{\mathbf{n}}_{3}\right]^{T}} \\
{\left[\cos \vartheta\left(\hat{\mathbf{n}}_{3} \times \hat{\mathbf{n}}_{1}\right)-\sin \vartheta\left(\hat{\mathbf{n}}_{1} \times \hat{\mathbf{n}}_{2}\right) \times \hat{\mathbf{n}}_{3}\right]^{T}} \\
{\left[\cos \vartheta \hat{\mathbf{n}}_{1} \times \hat{\mathbf{n}}_{2}-\sin \vartheta \hat{\mathbf{n}}_{1}\right]^{T}}
\end{array}\right],
$$

where the denominator $D$ is given by

$$
D \equiv\left(R\left(\hat{\mathbf{n}}_{2}, \vartheta\right) \hat{\mathbf{n}}_{1}\right) \cdot\left(\hat{\mathbf{n}}_{2} \times \hat{\mathbf{n}}_{3}\right)=-\sin (\vartheta-\lambda)
$$

\section{Discussion}

We have given a presentation of the Davenport angles in the same detail that is normally given to the standard twelve sets of Euler angles. The connection has been made between the Davenport angles and the 3-1-3 set of Euler angles, and this has been used to develop further relationships of the Davenport angles. A corollary of our work is that if a reorientation of a spacecraft is to be accomplished by rotational maneuvers about threc axes, then these must be Davenport axes. An application of the Davenport angles to attitude estimation is given in Reference 6.

\section{Acknowledgment}

The authors are grateful to R. V. F. Lopes for a careful reading of an early version of the manuscript.

\section{References}

[1] EULER, L. "De Motu Corporum Circa Punctum Fixum Mobilium," Commentatio 825 indicis ENESTROEMIANI, Opera posthuma, Vol. 2, 1862, pp. 43-62, also Leonhardi Euleri Opera Omnia, Series Secunda, Opera Mechanica Et Astronomica, Bascl, Vol. 9, 1968, pp. 413-441.

[2] MARKLEY, F. L. "Parametcrization of the Altitude," in WERTZ, J. R. (editor), Spacecraft Attitude Determination and Control, Kluwer Academic Publishers, Dordrccht, 1978, pp. $410-420$.

[3] SHUSTER, M. D. "A Survey of Attitude Representations," Joumal of the Astronautical Sciences, Vol. 41, No. 4, pp. 439-517, October-December 1993.

[4] JUNKINS, J. L. and TURNER, J. D. Optimal Spacecraft Rotational Maneuvers, Elsevier, Amsterdam, 1986.

[5] DAVENPORT, P. B. "Rotations about Nonorthogonal Axes," AIAA Joumal, Vol. 11, No. 6, June 1973, pp. 853-857.

[6] SHUSTER, M. D. "Deterministic Three-Axis Attitude Determination," submitted to the Joumal of the Astronautical Sciences. 